\title{
Articles
}

\section{Studies on Synthesis and Biological Activities of Novel Triazole Compounds Containing $N, N$-Dialkyldithiocarbamate}

\author{
L. Z. Xu, K. Jiao, S. S. Zhang, and S. P. Kuang \\ College of Chemistry and Molecule Engineering, Oingdao Lniversity of Science and Technology. Qingdao, 266042, China \\ Received January 17. 2002
}

\begin{abstract}
Nine titled compounds were synthesized and identified by IR, NMR. MS and elemental analysis. The results of the primary biological test show that all of these compounds have the activities of fungicide and plant growth regulator.
\end{abstract}

Key Words : $N, N$-Dialkyldithiocarbamate. Triazole. Biological

\section{Introduction}

As an important type of fungicides, triazole compounds are highly efficient. low poisonous and inward-absorbent. ${ }^{1-3}$ At present, the studies on triazole derivatives are mainly concentrated on compounds with triazole as the only active group. The report of triazole compounds that contain both triazole group and other active group in a single molecule has rarely been found. Some of the triazole compounds containing organophosphorous group have been proved to have good bioactivities. ${ }^{4}$ But triazole compounds containing $N, N$-dimethyldithiocarbamate have not been reported. $N, N$ dialkyldithiocarbamate has been known as broad-range fungicides and having different fungicidal mechanism with triazole compounds. In order to search for new triazole compounds with higher bioactivity, nime titled compounds were synthesized. The reactions are shown in Scheme I:

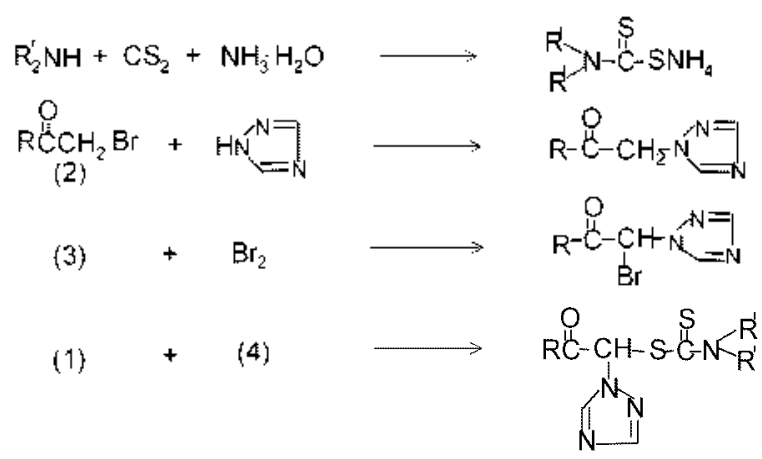

Scheme 1

\section{Experimental Section}

Materials and instruments: IR spectra were taken by Sclunadzu-IR- 435 Spectrophotometer ( $\mathrm{KBr}$ or liquid film).

Corresponding Author: e-mail: zhangshushapublic.qd.sd.cn

'Liangzhong Xul: Studying in Qungdao Ocean University for Ph.D.
${ }^{1} \mathrm{H}$ NMR was recorded with Bruker AC-P200Q nuclear magnetic resonance Spectrometer $\left(\mathrm{CDCl}_{3}\right.$ as solvent. TMS as internal standard). Mass spectra were taken by HP-5988A Spectrometer. Elemental analyses were perfomed using Yanaco-CHNCORDER MT-3 automatic elemental analyzer.

Preparation of intermediate (1): The intermediates (1) were prepared according to the literature report. ${ }^{5}$ Carbon disulfide, ammonium and dimetlyylamine were added together at around $10^{\circ} \mathrm{C}$, then the mixture were delydraled by refluxing with benzene. Three intermediates (1) were prepared in the same manner $\left(\mathrm{CH}_{3}: 79.2 \%, 86-87^{\circ} \mathrm{C} ; \mathrm{C}_{2} \mathrm{H}_{3}: 87.5 \%\right.$. 62$64{ }^{\circ} \mathrm{C} ; i-\mathrm{C}_{3} \mathrm{H}_{7}: 90.3 \% .46-48{ }^{\circ} \mathrm{C}$ ).

Preparation of intermediate (2): The intermediates (2) were prepared according to the literature report ${ }^{6}$ Aryl (or alkyl) etlyl ketone reacted with bromine in anlyydrous ether. Three intermediates (2) were prepared $\left(p-\mathrm{ClC}_{6} \mathrm{H}_{4}: 87.0 \%\right.$. $\left.66-67{ }^{\circ} \mathrm{C}: \mathrm{C}_{6} \mathrm{H}_{5}: 85.7 \%, 50-51{ }^{\circ} \mathrm{C}: \mathrm{Me}_{3} \mathrm{C}: 82.3 \% \cdot \mathrm{n}^{16}{ }^{16} 1.4663\right)$.

Preparation of intermediate (3): Three intermediates (3) were prepared by the reactions of 1.2.4-triazole and intermediate (2) in the presence of trietlylamine in acetone. $(p$ $\mathrm{ClC}_{6} \mathrm{H}_{4}: 80.2 \%, 150-152^{\circ} \mathrm{C}: \mathrm{C}_{6} \mathrm{H}_{5}: 81.0 \%, 117-119^{\circ} \mathrm{C} ; \mathrm{Me}_{3} \mathrm{C}$ : $74.5 \%, 62-644^{\circ} \mathrm{C}$ ).

Synthesis of S-[ $\alpha$-aryl-2-0xo-1-(1,2,4-triazol-1-yl)] ethyl$N, N$-dialkyldithiocar-bamate: To a $100 \mathrm{~mL}$ flask $15 \mathrm{mmol}$ intermediate (3), $25 \mathrm{~mL}$ acetic acid and $15 \mathrm{mmol}$ sodium acetate were added. Then $15 \mathrm{mmol}$ bromine was dropwise added with stirring at $15-25^{\circ} \mathrm{C}$. The reaction was maintained until the mixture was tumed into colorless or light yellow for about $2-3 \mathrm{~h}$. Then $50 \mathrm{~mL}$ water and $40 \mathrm{~mL}$ chloroform were added. Organic layer was successively washed with saturated sodium bicarbonate solution and brine, then dried over sodium sulfate and the chloroform solution containing about $15 \mathrm{mmol}$ intermediate (4) was filtrated into a $100 \mathrm{~mL}$ flask. Cooled with ice-ivater and $30 \mathrm{~mL}$ acetone solution of intermediate (1) were added under stirning and the mixture was stirred at around $0^{\circ} \mathrm{C}$ for $\mathrm{l} \mathrm{h}$. The solution was filtered, concentrated and purified by flash chromatograply (silica gel. $\mathrm{V}_{\text {ethyl ethanoate }}: \mathrm{V}_{\text {cyclohexane }}=5: 1$ ) to afford target conpounds (5). 
Table 1. The physical data and IR data of compounds $5 \mathbf{a}-5 \mathbf{i}$

\begin{tabular}{|c|c|c|c|c|c|c|c|c|c|}
\hline \multirow{2}{*}{$\begin{array}{c}\text { Compound } \\
\text { No. }\end{array}$} & \multirow{2}{*}{$\mathrm{R}^{\prime}$} & \multirow{2}{*}{$\mathrm{R}$} & \multirow{2}{*}{$\begin{array}{c}\text { Yield } \\
\%\end{array}$} & \multirow{2}{*}{ min. $\mathrm{p}^{/} \mathrm{C}$} & \multicolumn{3}{|c|}{ Elemental analysis (Calcd $/ \%$ ) } & \multicolumn{2}{|c|}{$\operatorname{IR}\left(\mathrm{v} / \mathrm{cm}^{-1}\right)$} \\
\hline & & & & & $\mathrm{C}$ & $\mathrm{H}$ & $\mathrm{N}$ & $v_{C=C 1}$ & $v_{C}=:$ \\
\hline $5 a$ & $\mathrm{CH}_{3}$ & $p$-Cl-Ph & 72.5 & $167-169$ & $46.01(45.81)$ & $3.70(3.84)$ & $16.37(16.44)$ & 1710 & 1511 \\
\hline $5 b$ & $\mathrm{C}_{2} \mathrm{H}_{5}$ & $p$-Cl-Ph & 70.4 & $110-112$ & $48.69(48.84)$ & $4.62(4.64)$ & $15.27(15.19)$ & 1690 & 1501 \\
\hline $5 \mathrm{c}$ & $i-\mathrm{C}_{3} \mathrm{H}_{-}$ & $p$-Cl-Ph & 69.5 & $66-68$ & $51.30(51.41)$ & $5.28(5.33)$ & $14.22(14.11)$ & 1699 & 1500 \\
\hline $5 d$ & $\mathrm{CH}_{3}$ & $\mathrm{Pl}$ & 71.2 & $125-127$ & $50.77(50.69)$ & $4.51(4.60)$ & $18.31(18.28)$ & 1683 & 1498 \\
\hline $5 e$ & $\mathrm{C}_{2} \mathrm{H}_{5}$ & Pli & 71.0 & $96-97$ & $63.62(53.87)$ & $5.38(5.42)$ & $16.91(16.75)$ & 1692 & 1501 \\
\hline $5 f$ & $i-\mathrm{C}_{3} \mathrm{H}_{-}$ & $\mathrm{Plh}$ & 68.3 & $52-53$ & $56.17(56.32)$ & $605(6.12)$ & $15.63(15.45)$ & 1675 & 1489 \\
\hline $5 \mathrm{~g}$ & $\mathrm{CH}_{3}$ & $\mathrm{Me}_{3} \mathrm{C}$ & 70.9 & $61-62$ & $45.99(46.13)$ & $6.32(6.33)$ & $19.51(19.56)$ & 1710 & 1490 \\
\hline $5 \mathrm{~h}$ & $\mathrm{C}_{2} \mathrm{H}_{5}$ & $\mathrm{Me}_{3} \mathrm{C}$ & 67.5 & $11 \mathrm{I}^{20} 1.5416$ & $49.37(49.65)$ & $7.02(7.05)$ & $17.91(17.82)$ & 1711 & 1498 \\
\hline $5 \mathbf{i}$ & $i-\mathrm{C}_{3} \mathrm{H}_{-}$ & $\mathrm{Me}_{3} \mathrm{C}$ & 62.1 & $11 \mathrm{I}^{18} 1.5221$ & $52.41(52.60)$ & $7.53(7.65)$ & $16.42(16.36)$ & 1710 & 1493 \\
\hline
\end{tabular}

Table 2. ${ }^{1} \mathrm{H}$ NMR $(\delta)$ and MS data of compounds $\mathbf{5 a - 5 i}$

\begin{tabular}{|c|c|c|c|c|c|}
\hline $\begin{array}{l}\text { Compound } \\
\text { No. }\end{array}$ & $\mathrm{R}^{\prime}$ & -SCH- & $\mathrm{R}$ & $\operatorname{Tr}^{2}$ & $\mathrm{MS}(\mathrm{m} / \mathrm{z})$ \\
\hline $5 a$ & $\begin{array}{l}3.37\left(\mathrm{~s}, 3 \mathrm{H}, \mathrm{CH}_{3}\right) \\
3.62\left(\mathrm{~s}, 3 \mathrm{H}, \mathrm{CH}_{3}\right)\end{array}$ & $7.90(\mathrm{~s}, \mathrm{lH})$ & $7.45-8.03\left(\mathrm{AB}_{3} 4 \mathrm{H}, \mathrm{C}_{6} \mathrm{H}_{4}\right)$ & $8.47-8.62(\mathrm{AB}, 2 \mathrm{H})$ & $\begin{array}{l}340\left(\mathrm{M}^{+}\right), 229,20 \\
139,133,120,88\end{array}$ \\
\hline $5 \mathbf{b}$ & $\begin{array}{l}1.25\left(\mathrm{~m}, 6 \mathrm{H}, 2 \mathrm{CH}_{3}\right) \\
3.62-4.05\left(\mathrm{~m}, 4 \mathrm{H}, 2 \mathrm{CH}_{2}\right)\end{array}$ & $7.88(\mathrm{~s}, \mathrm{lH})$ & $7.45-8.03\left(\mathrm{~m}, 4 \mathrm{H}, \mathrm{C}_{4} \mathrm{H}_{4}\right)$ & $8.45-8.60(\mathrm{AB}, 2 \mathrm{H})$ & \\
\hline $5 c$ & $\begin{array}{l}\mathrm{l} .42\left(\mathrm{~m}, 12 \mathrm{H}, 4 \mathrm{CH}_{3}\right) \\
4.51-4.72(\mathrm{~m}, 2 \mathrm{H}, 2 \mathrm{CH})\end{array}$ & $7.95(\mathrm{~s}, \mathrm{lH})$ & $7.45-8.10\left(\mathrm{~m}, 4 \mathrm{H}, \mathrm{C}_{4} \mathrm{H}_{4}\right)$ & $8.48-8.67(\mathrm{AB}, 2 \mathrm{H})$ & \\
\hline 5d & $\begin{array}{l}3.34\left(\mathrm{~s}, 3 \mathrm{H}, \mathrm{CH}_{3}\right) \\
3.38\left(\mathrm{~s}, 3 \mathrm{H}, \mathrm{CH}_{3}\right)\end{array}$ & $7.88(\mathrm{~s}, \mathrm{lH})$ & $7.45-8.02\left(\mathrm{~m}, 5 \mathrm{H}, \mathrm{C}_{4} \mathrm{H}_{4}\right)$ & $8.4-8.61(\mathrm{AB}, 2 \mathrm{H})$ & $\begin{array}{c}306\left(\mathrm{M}^{+}\right), 229,20 \\
133,120,88,77\end{array}$ \\
\hline $5 e$ & $\begin{array}{l}\mathrm{l} .26\left(\mathrm{~m}, 6 \mathrm{H}, 2 \mathrm{CH}_{3}\right) \\
3.64-4.29\left(\mathrm{~m}, 4 \mathrm{H}, 2 \mathrm{CH}_{2}\right)\end{array}$ & $7.89(\mathrm{~s}, \mathrm{lH})$ & $7.44-8.01\left(\mathrm{~m}, 5 \mathrm{H}, \mathrm{C}_{s} \mathrm{H}_{4}\right)$ & $8.47-8.60(\mathrm{AB}, 2 \mathrm{H})$ & \\
\hline $5 f$ & $\begin{array}{l}1.58\left(\mathrm{~m}, 12 \mathrm{H}, 4 \mathrm{CH}_{3}\right) \\
4.48-4.74(\mathrm{~m}, 2 \mathrm{H}, 2 \mathrm{CH})\end{array}$ & $7.96(\mathrm{~s}, \mathrm{lH})$ & $7.45-8.02\left(\mathrm{~m}, 5 \mathrm{H}, \mathrm{C}_{5} \mathrm{H}_{4}\right)$ & $8.50-8.74(\mathrm{AB}, 2 \mathrm{H})$ & \\
\hline $5 g$ & $\begin{array}{l}3.38\left(\mathrm{~s}, 3 \mathrm{H}, \mathrm{CH}_{3}\right) \\
3.33\left(\mathrm{~s}, 3 \mathrm{H}, \mathrm{CH}_{3}\right)\end{array}$ & $7.91(\mathrm{~s}, \mathrm{lH})$ & $1.22\left(\mathrm{~s}, 9 \mathrm{H}, 3 \mathrm{CH}_{3}\right)$ & $\begin{array}{l}7.96(\mathrm{~s}, 1 \mathrm{H}) \\
8.50(\mathrm{~s}, 1 \mathrm{H})\end{array}$ & $\begin{array}{c}286\left(\mathrm{M}^{+}\right), 229,20 \\
120,88,57\end{array}$ \\
\hline $5 \mathbf{h}$ & $\begin{array}{l}1.28\left(\mathrm{~m}, 6 \mathrm{H}, 2 \mathrm{CH}_{3}\right) \\
3.65-4.00\left(\mathrm{~m}, 4 \mathrm{H}, 2 \mathrm{CH}_{2}\right)\end{array}$ & $7.92(\mathrm{~s}, \mathrm{lH})$ & $1.20\left(\mathrm{~s}, 9 \mathrm{H}, 3 \mathrm{CH}_{3}\right)$ & $\begin{array}{l}7.96(\mathrm{~s}, 1 \mathrm{H}) \\
8.49(\mathrm{~s}, 1 \mathrm{H})\end{array}$ & \\
\hline $\mathbf{5 i}$ & $\begin{array}{l}1.30\left(\mathrm{~m}, 12 \mathrm{H}, 4 \mathrm{CH}_{2}\right) \\
4.47-4.69(\mathrm{~m}, 2 \mathrm{H}, 2 \mathrm{CH})\end{array}$ & $7.94(\mathrm{~s}, \mathrm{lH})$ & $1.20\left(\mathrm{~s}, 9 \mathrm{H}, 3 \mathrm{CH}_{3}\right)$ & $\begin{array}{l}8.01(\mathrm{~s}, 1 \mathrm{H}) \\
8.56(\mathrm{~s}, 1 \mathrm{H})\end{array}$ & \\
\hline
\end{tabular}

"T $\mathrm{T}=1.2 .4$-triazol-1-yl

\section{Results and Discussion}

Synthesis of intermediate (1) and target compounds (4): After a water solution of intermediate (1) being prepared according to literature. ${ }^{5}$ the yellow crystal of intermediate (1) can be obtained by dehydration in benzene as reflux agent. When toluene was used as reflux agent instead of benzene. the color of the crystal became dark and the yield was reduced. The intermediate (t) was sy'nthesized according to the literature. ${ }^{7}$ We got two products. One is intermediate (t), another is the dibromo-substituted product confirmed by ${ }^{l} \mathrm{H}$ NMR spectrum and elemental analysis. The reaction between intermediate $(t)$ and intermediate (1) can be performed directly in acetone

Spectrum characterization of compounds $\mathbf{5 a - 5 j}$ : The experimental results with IR. ${ }^{1} \mathrm{H}$ NMR and MS data are shown in Tables 1 and Table 2 . The measured values in the elemental analy'sis are in consistence with the corresponding calculated ones. All compounds (5a-5i) show very strong absorption bands in IR spectra at $1711 \mathrm{~cm}^{-1}-1675 \mathrm{~cm}^{-1}$ and $1489 \mathrm{~cm}^{-1}-1511 \mathrm{~cm}^{-1}$. Which are characteristic absorption bands for $v_{C}=0$ and $v_{C}=k$. respectively:

The ${ }^{\mathrm{l}} \mathrm{H}$ NMR data for compounds (5a-5i) are as predicted except for two protons on triazole ring. The chemical shifts for triazole ring protons are affected by $\mathrm{R}$ group. When $\mathrm{R}$ is $p$ - $\mathrm{ClC}_{6} \mathrm{H}_{4}$ (or $\mathrm{C}_{6} \mathrm{H}_{3}$ ). the chemical shifts for the two protons are very close at $\delta=8.44-8.74$ and couple with each other into $\mathrm{AB}$ peak. When $\mathrm{R}$ is $\mathrm{Me}_{3} \mathrm{C}$. their chemical shifts are at $\delta$ $=8.00$ and $\delta=8.50$. respectively.

Biological activity: Each of the target compounds has the fungicidal activity (Table 3). They exhibit better efficiency towards $P$. asparagi on the whole. such as $\mathbf{5 g}$ and $\mathbf{5 h}$. The inhibiting rates reach $100 \%$ at $50 \mu \mathrm{g} / \mathrm{mL}$. When $\mathrm{R}$ is tenbutyl group, the compounds have better comprehensive inhibiting rates than that $\mathrm{R}$ is aryl group. As far as $\mathrm{R}^{\prime}$ is concerned, inluibiting activities of the compounds $\left(\mathrm{R}^{\prime}=\mathrm{CH}_{3}\right)$ are higher than the compounds $\left(\mathrm{R}^{\prime}=\mathrm{CH}_{2} \mathrm{CH}_{3}\right)$. The activity order for $\mathrm{R}^{\prime}$ is: $\mathrm{CH}_{3}>\mathrm{CH}_{2} \mathrm{CH}_{2}>i_{i}-\mathrm{C}_{3} \mathrm{H}_{7}$. All the nine target 
Table 3. The fungicidal and plant growth regulator activities of compounds 5

\begin{tabular}{|c|c|c|c|c|c|c|c|c|}
\hline \multirow{2}{*}{$\begin{array}{l}\text { Compound } \\
\text { No. }\end{array}$} & \multicolumn{5}{|c|}{ Fungicidal activities $(\mathrm{c}=0.005 \%$, inlibition $\%)$} & \multicolumn{3}{|c|}{ Plant grouth regulator activities $(\mathrm{c}=0.0001 \%, \%)$} \\
\hline & P. zeae & A. solani & Pasparagi & P piricola & C. Arachidicala & $\begin{array}{l}\text { wheat coleoptile } \\
\text { elougation }\end{array}$ & $\begin{array}{l}\text { Rooting of cumcumber } \\
\text { cotyledon }\end{array}$ & $\begin{array}{c}\text { Rape hypocotyl } \\
\text { inhibition }\end{array}$ \\
\hline $5 a$ & 26.5 & 78.9 & 27.3 & 38.5 & 45.5 & -17.6 & +22.2 & -5.3 \\
\hline 5b & 50.4 & 63.2 & 72.7 & 50.0 & 45.5 & -12.0 & +72.2 & -30.1 \\
\hline $5 c$ & 17.6 & 5.3 & 45.5 & 23.1 & 18.2 & -10.2 & +17.8 & +0.9 \\
\hline $5 d$ & 32.4 & 97.4 & 90.9 & 15.4 & 81.8 & -10.2 & +233.3 & -30.1 \\
\hline 5e & 32.4 & 73.7 & 63.6 & 30.8 & 50.4 & -16.7 & +116.6 & +5.3 \\
\hline sf & 23.5 & 0 & 80.0 & 15.4 & 36.4 & -9.3 & +233.3 & +4.4 \\
\hline $5 \mathrm{~g}$ & 66.5 & 64.5 & 100.0 & 53.8 & 18.2 & -14.8 & +72.2 & -0.9 \\
\hline $5 \mathrm{~h}$ & 44.9 & 36.8 & 100.0 & 46.9 & 54.5 & -16.7 & +133.3 & +3.5 \\
\hline $5 i$ & 29.4 & 26.3 & 81.8 & 34.6 & 13.6 & -18.52 & +133.3 & +0.9 \\
\hline
\end{tabular}

compounds have plant growth regulating activity. They show inhibiting activity towards wheat coleoptile elougation. The inhibiting rates reach $9.26 \%-18.52 \%$. The promoting activity towards Rooting cumcumber cotyledon. the promoting rates reach $17.78 \%-233.3 \%$. Most of the compounds possess lower inhibiting activity towards Rape hypocony. The highest inhibiting rate is $30.1 \%$.

Acknow ledgment. This work was supported by the NSFC project 20275020 . China

\section{References}

1. Shi. Y.-N.: Lu. Y.-C.: Fang. T.-X. Chemical d. Chinese Lnwersities 1995. $16(11) .1710-1713$.

2. Elbe. H. L.: Buechel. K. H.: Luerssen, K. ef al. Ger Offen. 3144 670. 1983

3. Anderson. N. H. Phoceeding of 5 h Intenational Congress of Pesticide Chemistry (ILPAC): Kyoto Japan 1982: p 345.

4. Shi. Y.-N.: Xu. L.-Z.: Fang. J.-X. Chenical J. Chimese Lniversities 1991. $9(12) .1190-1193$.

5. Gracia Albert. J. LS. Patent. $2229562,1941$.

6. Hill. G. H.: Kropa. L. E. J. Am. Chem. Soc. 1953. $55,2509$.

7. Holmwood. G.; Oeckl, S.; Stetter, J. Ger. Offen. 2937595.1981. 